Bull. Austral. Math. Soc.

VoL. 49 (1994) [69-79]

\title{
OSCILLATION AND NONOSCILLATION OF A DELAY DIFFERENTIAL EQUATION
}

\author{
Chunhai Kou, Weiping Yan and Jurang YaN
}

In this paper, some necessary and sufficient conditions for oscillation of a first order delay differential equation with oscillating coefficients of the form

$$
x^{\prime}(t)+\sum_{i=1}^{n} p_{i}(t) x\left(t-\tau_{i}(t)\right)=0
$$

are established. Several applications of our results improve and generalise some of the known results in the literature.

\section{Introduction}

The oscillatory behaviour of first order delay differential equation has been studied by many authors. In particular, several authors have studied the oscillations caused by deviating arguments. Besides its theoretical interest, this question is important from the viewpoint of practical applications. For some contributions in this area see [2] and references cited therein. Most of the known results, however, deal with equations having coefficients with fixed sign. The case where the coefficients are oscillating has been considered in only a few papers, for example, $[5,8]$ and $[9]$.

Let us consider the first order differential equation with oscillating coefficients

$$
x^{\prime}(t)+\sum_{i=1}^{n} p_{i}(t) x\left(t-\tau_{i}(t)\right)=0
$$

where $p_{i}, \tau_{i}, i=1,2, \cdots, n$, are continuous functions on the interval $\left[t_{0}, \infty\right)$ and $\lim _{t \rightarrow \infty}\left(t-\tau_{i}(t)\right)=\infty, \quad i=1,2, \cdots, n$. If $T \geqslant t_{0}$, by a solution on $[T, \infty)$ of equation (1) we mean a real-valued function $x(t)$ defined on $[T, \infty)$, which is differentiable on $[T, \infty)$ and satisfies $x^{\prime}(t)+\sum_{i=1}^{n} p_{i}(t) \tilde{x}\left(t-\tau_{i}(t)\right)=0$ for $t \geqslant T$, where $\tilde{x}$ is a continuous extension of $x(t)$ on $\left[T^{*}, \infty\right)$ with $T^{*}=\min _{1 \leqslant i \leqslant n}\left\{\inf \left(t-\tau_{i}(t)\right)\right\}$. A solution of equation (1) is said to be oscillatory if it has arbitrarily large zeros, and otherwise it is called non-oscillatory.

Received 4th February, 1993

Copyright Clearance Centre, Inc. Serial-fee code: 0004-9729/94 \$A2.00+0.00. 
We say that the conditions (C) hold if the following conditions are fulfilled:

$\left(C_{1}\right) \quad \tau_{i}(t), i=1,2, \cdots, n$ are continuous on $\left[t_{0}, \infty\right)$, and there exist two constants $\alpha$ and $\beta$ so that $0<\alpha \leqslant \tau_{n}(t) \leqslant \tau_{n-1}(t) \leqslant \cdots \leqslant \tau_{1}(t) \leqslant \beta$.

$\left(C_{2}\right) \quad p_{i}(t), i=1,2, \cdots, n$, are continuous on $\left[t_{0}, \infty\right)$, and for each $m, m=$ $1,2, \cdots, n, \quad \sum_{i=1}^{m} p_{i}(t) \geqslant 0 ;$ Furthermore, there exists a sequence of intervals $\left\{\left[a_{k}, b_{k}\right]\right\}$ with $b_{k}-a_{k} \geqslant \beta$ such that $p_{i}(t) \geqslant 0, i=1,2, \cdots, n, t \in \bigcup_{k=1}^{\infty}\left(a_{k}, b_{k}\right)$.

Recently, under the conditions (C) Yongchao Chen [1] studied equation (1) with $\tau_{i}(t) \equiv \tau_{i}$ (constants), $i=1,2, \cdots, n$ and gave the following main results.

Theorem A. Assume that the conditions (C) hold and

$$
\begin{aligned}
& \liminf _{t \rightarrow \infty} \int_{t-\tau_{n}^{*} / 2}^{t} \sum_{i=1}^{n} p_{i}(s) d s>0, \\
& \lim _{t \rightarrow \infty} \text { inf } \int_{t-\tau_{n}^{*}}^{t} \sum_{i=1}^{n} p_{i}(s) d s>\frac{1}{e} .
\end{aligned}
$$

Then all solutions of equation (1) with $r_{i}(t) \equiv r_{i}$ (constants), $i=1,2, \cdots, n$, are oscillatory.

In this paper, by using a new technique we establish necessary and sufficient conditions, and explicit sufficient conditions for all solutions of equation (1) to be oscillatory. We also give conditions, under which equation (1) has at least one non-oscillatory solution and estimate of the asymptotic behaviour of the non-oscillatory solution.

\section{MaIN TheOREMS}

The following two lemmas are basic for all later discussions and Lemma 1 is a extension of Lemma in [1].

LEMma 1. Assume that the conditions (C) hold. Let $x(t)$ be an eventually positive solution of equation (1). Then for sufficiently large $t, x(t)$ is eventually nonincreasing and

$$
\left(\sum_{i=1}^{n} p_{i}(t)\right) x\left(t-\tau_{n}(t)\right) \leqslant \sum_{i=1}^{n} p_{i}(t) x\left(t-\tau_{i}(t)\right) .
$$

Proof: Obviously, there exists $T \geqslant t_{0}$ so that

$$
x\left(t-\tau_{i}(t)\right)>0, \quad i=1,2, \cdots, n, \quad \text { for every } t \geqslant T .
$$

From (C), there exists $t_{1} \geqslant T$ such that $p_{i}(t) \geqslant 0, i=1,2, \cdots, n$, on $\left[t_{1}, t_{1}+\beta\right]$. Thus

$$
x^{\prime}(t)=-\sum_{i=1}^{n} p_{i}(t) x\left(t-\tau_{i}(t)\right) \leqslant 0, \quad \text { for all } t \in\left[t_{1}, t_{1}+\beta\right]
$$


which implies that $x(t)$ is non-increasing on $\left[t_{1}, t_{1}+\beta\right]$. We now claim that $x(t)$ is also non-increasing on $\left[t_{1}, t_{1}+\beta+\alpha\right]$. Indeed, from $\left(C_{1}\right)$ and (3), for every $t \in$ $\left[t_{1}+\beta, t_{1}+\beta+\alpha\right]$, we have that $t-\tau_{i}(t) \in\left[t_{1}, t_{1}+\beta\right], \quad i=1,2, \cdots, n$, and

$$
x\left(t-\tau_{1}(t)\right) \geqslant x\left(t-\tau_{2}(t)\right) \geqslant \cdots \geqslant\left(t-\tau_{n}(t)\right) .
$$

By using the condition $\left(C_{2}\right),(4)$ yields the inequality

$$
\begin{aligned}
x^{\prime}(t) & =-\sum_{i=1}^{n} p_{i}(t) x\left(t-\tau_{i}(t)\right) \\
& \leqslant-p_{1}(t) x\left(t-\tau_{2}(t)\right)-\sum_{i=2}^{n} p_{i}(t) x\left(t-\tau_{i}(t)\right) \\
& \leqslant-\left(p_{1}(t)+p_{2}(t)\right) x\left(t-\tau_{2}(t)\right)-\sum_{i=3}^{n} p_{i}(t) x\left(t-\tau_{i}(t)\right) \leqslant \cdots \cdots \\
& \leqslant-\sum_{i=1}^{n} p_{i}(t) x\left(t-\tau_{n}(t)\right) \leqslant 0, \quad \text { for } \quad t \in\left[t_{1}+\beta, t_{1}+\beta+\alpha\right] .
\end{aligned}
$$

This means $x(t)$ is also non-increasing on $\left[t_{1}+\beta, t_{1}+\beta+\alpha\right]$. By repeating the same procedure $k \geqslant 1$ times we are led to the following inequality:

$$
\begin{aligned}
x^{\prime}(t) & =-\sum_{i=1}^{n} p_{i}(t) x\left(t-\tau_{i}(t)\right) \\
& \leqslant-\left(\sum_{i=1}^{n} p_{i}(t)\right) x\left(t-\tau_{n}(t)\right), \text { for } t \in\left[t_{1}+\beta, t_{1}+\beta+k \alpha\right] .
\end{aligned}
$$

Since $k$ is aribitrary, the above inequality implies that for all $t \geqslant t_{1}, x(t)$ is nonincreasing and

$$
\left(\sum_{i=1}^{n} p_{i}(t)\right) x\left(t-\tau_{n}(t)\right) \leqslant \sum_{i=1}^{n} p_{i}(t) x\left(t-\tau_{i}(t)\right) .
$$

The proof of Lemma 1 is complete.

In the sequel, unless otherwise specified, for sufficiently large $T \geqslant t_{0}$, we define

$$
T_{0}=\min _{1 \leqslant i \leqslant n}\left\{\inf _{t \geqslant T}\left(t-\tau_{i}(t)\right)\right\} .
$$

Lemma 2. Assume that the conditions (C) hold. Then for sufficiently large $T \geqslant$ $t_{0}$, the differential inequality

$$
x^{\prime}(t)+\sum_{i=1}^{n} p_{i}(t) x\left(t-\tau_{i}(t)\right) \leqslant 0, \quad t \geqslant T
$$


has a positive continuous differentiable solution on $[T, \infty)$ if and only if the integral inequality

$$
\sum_{i=1}^{n} p_{i}(t) \exp \left(\int_{t-r_{i}(t)}^{t} u(s) d s\right) \leqslant u(t), \quad t \geqslant T
$$

has a non-negative solution on $[T, \infty)$.

ProOf: Let $x(t)$ be a positive solution on $[T, \infty)$ of equation $(1)$ and for all $t \geqslant T_{0}$, $x\left(t-\tau_{i}(t)\right)>0, \quad i=1,2, \cdots, n$, where $T_{0}$ is as defined by (5). Set

$$
u(t)=-\frac{x^{\prime}(t)}{x(t)} \text { for } t \geqslant T_{0}
$$

From Lemma 1, without loss of generality, for $t \geqslant T_{0}$ we can suppose that $u(t) \geqslant 0$. By integrating both sides of (8) from $T$ to $t$ we obtain

$$
x(t)=x(T) \exp \left(-\int_{T}^{t} u(s) d s\right)
$$

Substituting (9) into (6), we can see that $u(t)$ is a non-negative continuous solution of (7) on $[T, \infty)$.

Conversely, let $u(t)$ be a non-negative continuous solution of $(7)$ on $[T, \infty)$. It is easy to verify that $x(t)=\exp \left(-\int_{T}^{t} u(s) d s\right)$ is a positive continuous differentiable solution of $(6)$ on $[T, \infty)$. The proof is complete.

With (1) and for every $T \geqslant t_{0}$, we introduce the sequence of functions:

$$
u_{0}(t)=0 \quad \text { for } \quad t \geqslant T
$$

and for $k=1,2, \cdots$.

$$
u_{k}(t)= \begin{cases}\sum_{i=1}^{n} p_{i}(t) \exp \left(\int_{t-\tau_{i}(t)}^{t} u_{k-1}(s) d s\right) & \text { for } t \geqslant T, \\ 0 & \text { for } T_{0} \leqslant t<T,\end{cases}
$$

where $T_{0}$ is as defined by (5). It is obvious that $u_{k}(t), k=1,2, \cdots$, is continuous on $\left[T_{0}, T\right)$ and $\left(T_{1}, \infty\right)$, respectively.

Since $u_{0}(t)=0, t \geqslant T_{0}, u_{1}(t)=\sum_{i=1}^{n} p_{i}(t) \geqslant 0, t \geqslant T$, and $u_{1}(t) \geqslant 0, t \geqslant T_{0}$, 
hence $u_{1}(t) \geqslant u_{0}(t)$ for $t \geqslant T_{0}$. From (C) and (10) we have

$$
\begin{aligned}
u_{2}(t) & \geqslant p_{1}(t) \exp \left(\int_{t-\tau_{2}(t)}^{t} u_{1}(s) d s\right)+\sum_{i=2}^{n} p_{i}(t) \exp \left(\int_{t-\tau_{i}(t)}^{t} u_{1}(s) d s\right) \\
& \geqslant\left(p_{1}(t)+p_{2}(t)\right) \exp \left(\int_{t-\tau_{2}(t)}^{t} u_{1}(s) d s\right)+\sum_{i=3}^{n} p_{i}(t) \exp \left(\int_{t-\tau_{i}(t)}^{t} u_{1}(s) d s\right) \\
& \geqslant \cdots \geqslant \sum_{i=1}^{n} p_{i}(t) \exp \left(\int_{t-\tau_{n}(t)}^{t} u_{1}(s) d s\right) \\
& \geqslant \sum_{i=1}^{n} p_{i}(t)=u_{1}(t), \quad \text { for } t \geqslant T,
\end{aligned}
$$

which means that $u_{2}(t) \geqslant u_{1}(t)$ for all $t \geqslant T_{0}$. By induction it is easy to see that for $t \geqslant T_{0}$

$$
u_{k+i}(t) \geqslant u_{k}(t) \geqslant 0, \quad k=1,2, \cdots
$$

Now we are ready to establish the following theorem.

Theorem 1. Assume that the conditions (C) hold. Then the following statements are equivalent:

(a) Equation (1) has a non-oscillatory solution.

(b) The delay differential inequality (6) has an eventually positive solution.

(c) There exists a sufficiently large $T \geqslant t_{0}$ such that the sequence $\left\{u_{k}(t)\right\}$ which is defined by (10) converges pointwise to a finite limit for each $t \geqslant T_{0}$.

ProOF: (a) $\Rightarrow(b)$. Let $x(t)$ be a non-oscillatory solution of equation (1). As the negative of a solution of equation (1) is also a solution of the same equation, we can assume that $x(t)$ is eventually positive. Clearly, $x(t)$ is also an eventually positive solution of (6).

(b) $\Rightarrow(c)$. Assume that (6) has an eventually positive solution $x(t)$ and there exists $T^{*} \geqslant t_{0}$ such that $x(t)>0, t \geqslant T^{*}$. From (5) we can choose $T$ such that $T_{0} \geqslant T^{*}$. Set $u(t)=-x^{\prime}(t) / x(t)$. By using Lemma 2, it follows that the inequality (7) has a non-negative solution $u(t)$ which is defined in $\left[T_{0}, \infty\right)$. Consider the sequence 
(10). Noting $u(t) \geqslant u_{0}(t), t \geqslant T_{0}$ and using (10) and (C), we find that

$$
\begin{aligned}
u_{1}(t)= & \sum_{i=1}^{n} p_{i}(t) \leqslant\left(\sum_{i=1}^{n} p_{i}(t)\right) \exp \left(\int_{t-\tau_{n}(t)}^{t} u(s) d s\right) \\
\leqslant & \left(\sum_{i=1}^{n-1} p_{i}(t)\right) \exp \left(\int_{t-\tau_{n-1}(t)}^{t} u(s) d s\right)+p_{n}(t) \exp \left(\int_{t-\tau_{n}(t)}^{t} u(s) d s\right) \\
\leqslant & \left(\sum_{i=1}^{n-2} p_{i}(t)\right) \exp \left(\int_{t-\tau_{n-2}(t)}^{t} u(s) d s\right)+p_{n-1}(t) \exp \left(\int_{t-\tau_{n-1}(t)}^{t} u(s) d s\right) \\
& +p_{n}(t) \exp \left(\int_{t-\tau_{n}(t)}^{t} u(s) d s\right) \leqslant \cdots \leqslant \sum_{i=1}^{n} p_{i}(t) \exp \left(\int_{t-\tau_{i}(t)}^{t} u(s) d s\right) \\
\leqslant & u(t), \quad \text { for } t \geqslant T_{0} .
\end{aligned}
$$

So, $u_{1}(t) \leqslant u(t), t \geqslant T_{0}$. By a simple induction, we can show that

$$
0 \leqslant u_{k}(t) \leqslant u(t), \quad k=0,1,2, \cdots,
$$

for $t \geqslant T_{0}$. Then from (11) it follows that

$$
0 \leqslant u_{k}(t) \leqslant u_{k+1}(t) \leqslant u(t), \quad k=0,1,2, \cdots
$$

for $t \geqslant T_{0}$. Therefore, the sequence $\left\{u_{k}(t)\right\}$ converges pointwise to a finite limit for all $t \geqslant T_{0}$.

(c) $\Rightarrow(\mathrm{a})$. Set $\tilde{u}(t)=\lim _{k \rightarrow \infty} u_{k}(t), \quad t \geqslant T_{0}$. By Lebesgue's monotone convergence theorem, $\tilde{u}(t)$ satisfies

$$
\tilde{u}(t)= \begin{cases}\sum_{i=1}^{n} p_{i}(t) \exp \left(\int_{t-\tau_{i}(t)}^{t} \tilde{u}(s) d s\right), & t \geqslant T, \\ 0, & T_{0} \leqslant t<T\end{cases}
$$

which implies that $\tilde{u}(t)$ is a continuous solution of the integral equation

$$
u(t)=\sum_{i=1}^{n} p_{i}(t) \exp \left(\int_{t-\tau_{i}(t)}^{t} u(s) d s\right)
$$

Let $x(t)=\exp \left(-\int_{T}^{t} \tilde{u}(s) d s\right)$. Then it is easy to verify that $x(t)$ is a positive solution of equation (1).

The proof of Theorem 1 is complete.

The next result is an immediate consequence of Theorem 1. 
Theorem 2. Assume that the conditions (C) hold. Then the following statements are equivalent.

$\left(a_{1}\right)$ All solutions of equation (1) are oscillatory.

$\left(b_{1}\right)$ The inequality (6) has no eventually positive solutions.

$\left(c_{1}\right)$ For every large $T \geqslant t_{0}$ there exists $t^{*} \geqslant T$ such that the sequence (10) satisfy

$$
\lim _{k \rightarrow \infty} u_{k}\left(t^{*}\right)=\infty
$$

\section{APPLICATIONS OF MAIN THEOREM}

In this section we apply the above main theorems to give some sufficient conditions for equation (1) to have at least one non-oscillatory solution and for all solutions of equation (1) to be oscillatory.

Theorem 3. Assume that the conditions (C) hold, and there exists sufficiently large $T \geqslant t_{0}$ such that for all $t \geqslant T$,

$$
\int_{t-n_{1}(t)}^{t} \sum_{i=1}^{n} p_{i}^{+}(s) d s \leqslant \frac{1}{e},
$$

where $p_{i}^{+}(t)=\max \left\{p_{i}(t), 0\right\}$. Then equation (1) has a non-oscillatory solution $x(t)$ with

$$
\exp \left(-e \int_{T}^{t} \sum_{i=1}^{n} p_{i}^{+}(s) d s\right) \leqslant x(t) \leqslant \exp \left(-\int_{T}^{t} \sum_{i=1}^{n} p_{i}(s) d s\right)
$$

for all $t \geqslant T$.

Proof: Consider the sequence (10) on $\left(T_{0}, \infty\right)$, where $T_{0}$ is as defined by (5). From $(10)$ and $\left(C_{2}\right)$ it follows that $0 \leqslant u_{1}(t)=\sum_{i=1}^{n} p_{i}(t) \leqslant e \sum_{i=1}^{n} p_{i}^{+}(t), \quad t \geqslant T$. Thus $0 \leqslant u_{1}(t) \leqslant e \sum_{i=1}^{n} p_{i}^{+}(t), \quad t \geqslant T_{0}$. In view of (10) and (13) we obtain

$$
u_{2}(t) \leqslant \sum_{i=1}^{n} p_{i}^{+}(t) \exp \left(\int_{t-\tau_{1}(t)}^{t} u_{1}(s) d s\right) \leqslant e \sum_{i=1}^{n} p_{i}^{+}(t), \quad t \geqslant T
$$

Hence $u_{2}(t) \leqslant e \sum_{i=1}^{n} p_{i}^{+}(t), t \geqslant T_{0}$. By (10), (13) and a simple induction, it is easy to show that

$$
u_{k}(t) \leqslant e \sum_{i=1}^{n} p_{i}^{+}(t), \quad t \geqslant T_{0}, k=0,1,2, \cdots
$$


Thus, by applying Theorem 1, we know that equation (1) has a non-oscillatory solution. Let us now prove (14). Set

$$
\tilde{u}(t)=\lim _{k \rightarrow \infty} u_{k}(t), \quad \text { pointwise on }[T, \infty),
$$

then $\tilde{u}(t)$ satisfies (12). Then it is easy to verify that

$$
x(t)=\exp \left(-\int_{T}^{t} \tilde{u}(s) d s\right)
$$

is a positive solution on $[T, \infty)$ of equation (1). From (10) and (15), we have that $\sum_{i=1}^{n} p_{i}(t) \leqslant \tilde{u}(t) \leqslant e \sum_{i=1}^{n} p_{i}^{+}(t), t \geqslant T$, which means that the estimate (14) hold. The proof is complete.

THEOREM 4. Assume that the conditions (C) hold and for sufficiently large $T$ there exists $\lambda>0$ such that

$$
\sup _{t \geqslant T}\left\{\frac{1}{\lambda} \sum_{i=1}^{n} p_{i}^{+}(t) \exp \left(\lambda \tau_{i}(t)\right)\right\} \leqslant 1,
$$

where $p_{i}^{+}(t)=\max \left\{p_{i}(t), 0\right\}$. Then equation (1) has a non-oscillatory solution.

Proof: Consider the sequence $(10)$ on $\left[T_{0}, \infty\right)$, where $T_{0}$ is as defined by (5). $u_{1}(t)=\sum_{i=1}^{n} p_{i}(t) \leqslant \lambda_{1}$ where $\lambda_{1}=\sup _{t \geqslant T} \sum_{i=1}^{n} p_{i}^{+}(t)$. Since $u_{1}(t)=0, T_{0} \leqslant t<T, \quad u_{1}(t) \leqslant$ $\lambda_{1}, t \geqslant T_{0}$. Also $u_{2}(t) \leqslant \sum_{i=1}^{n} p_{i}^{+}(t) \exp \left(\int_{t-\tau_{i}(t)}^{t} u_{1}(s) d s\right) \leqslant \sum_{i=1}^{n} p_{i}^{+}(t) \exp \left(\lambda_{1} \tau_{i}(t)\right) \leqslant \lambda_{2}$ where $\lambda_{2}=\sup _{t \geqslant T}\left\{\sum_{i=1}^{n} p_{i}^{+}(t) \exp \left(\lambda_{1} \tau_{i}(t)\right)\right\}$. Obviously, $\lambda_{1} \leqslant \lambda_{2}$. By induction it is easy to see that

$$
\begin{gathered}
u_{k+1}(t) \leqslant \sum_{i=1}^{n} p_{i}^{+}(t) \exp \left(\lambda_{k} \tau_{i}(t)\right) \leqslant \lambda_{k+1}, \\
\lambda_{k+1}=\sup _{t \geqslant T}\left\{\sum_{i=1}^{n} p_{i}^{+}(t) \exp \left(\lambda_{k} \tau_{i}(t)\right)\right\},
\end{gathered}
$$

and $\lambda_{k} \leqslant \lambda_{k+1}$. By using (17), we derive that

$$
\lambda_{1} \leqslant \sum_{i=1}^{n} p_{i}^{+}(t) \exp \left(\lambda \tau_{i}(t)\right) \leqslant \lambda, \quad t \geqslant T .
$$

Hence, it is easy to show that $\lambda_{k} \leqslant \lambda, k=1,2, \cdots$, and $u_{k}(t) \leqslant \lambda, t \geqslant T$. By applying Theorem 1, we conclude that equation (1) has a non-oscillatory solution. The proof is complete.

Now, we establish the following oscillation comparison theorem. 
THEOREM 5. Assume that the condition (C) hold and all solutions of the delay differential equation

$$
x^{\prime}(t)+\sum_{i=1}^{n} p_{i}(t) x\left(t-\tau_{n}(t)\right)=0
$$

are oscillatory. Then all solutions of equation (1) are also oscillatory.

Proof: Suppose that equation (1) has a non-oscillatory solution $x(t)$. As the opposite of a solution of equation (1) is also a solution of the same equation, we may suppose that $x(t)>0$ for all $t \geqslant t_{1} \geqslant t_{0}$. By Lemma 1 , for all sufficiently large $t$, we have

$$
x^{\prime}(t)+\sum_{i=1}^{n} p_{i}(t) x\left(t-\tau_{n}(t)\right) \leqslant 0 .
$$

From Theorem 2 of [6], it follows that equation (18) has an eventually positive solution. This is a contradiction. The proof of the theorem is complete.

Combining Theorem 5 with known oscillation criteria for equation (18) (see $[2,3$, $4,7])$ we may obtain various results for oscillation of all solutions of equation (1).

Corollary 1. Assume that the conditions (C) hold and each one of the following conditions is satisfied,

$$
\begin{aligned}
& \text { (i) } \quad \lim _{t \rightarrow \infty} \inf \int_{t-\tau_{n}(t)}^{t} \sum_{i=1}^{n} p_{i}(s) d s>\frac{1}{e} \text {, } \\
& \text { (ii) } \quad \lim _{t \rightarrow \infty} \inf \left\{\tau_{n}(t) \sum_{i=1}^{n} p_{i}(t) d t\right\}>\frac{1}{e} \text {. }
\end{aligned}
$$

Then all solitions of equation (1) are oscillatory.

COROLlary 2. Assume that the conditions $(C)$ hold and the function $t-\tau_{n}(t)$ is non-decreasing. Moreover, assume that each one of the following conditions is satisfied:

$$
\begin{aligned}
\text { (i) } & \lim _{t \rightarrow \infty} \sin \int_{t-\tau_{n}(t)}^{t} \sum_{i=1}^{n} p_{i}(s) d s>1, \\
\text { (ii) } & \lim _{t \rightarrow \infty} \text { inf } \int_{t-\tau_{n}(t)}^{t} \sum_{i=1}^{n} p_{i}(s) d s=\alpha \leqslant \frac{1}{e}, \text { and } \\
& \lim _{t \rightarrow \infty} \inf \int_{t-\tau_{n}(t)}^{t} \sum_{i=1}^{n} p_{i}(s) d s>\frac{\ln \lambda_{\alpha}+1}{\lambda_{\alpha}},
\end{aligned}
$$

where $\lambda_{\alpha}$ is the smaller solution of the equation $\lambda=e^{\alpha \lambda}$. Then all solutions of equation (1) are oscillatory. 
REMARK. Obviously, the condition (i) of Corollary 1 improves and generalises Theorem A.

EXAMPle. Consider the differential equation

$$
x^{\prime}(t)+a x\left(t-\tau_{1}\right)+b \sin t x\left(t-\tau_{2}\right)+c \sin \frac{t}{2} x\left(t-\tau_{3}\right)=0, \quad t \geqslant 0
$$

where $a, b, c, \tau_{1}, \tau_{2}$, and $\tau_{3}$ are positive constants with $a \geqslant b+c$ and $\tau_{3}<\tau_{2}<\tau_{1} \leqslant \pi$. It is easy to check that equation (19) satisfies the condition (C). If

$$
\int_{t-\tau_{1}}^{t}\left[a+b(\sin s)^{+}+c\left(\sin \frac{s}{2}\right)^{+}\right] d s \leqslant \frac{1}{e},
$$

then, by Theorem 3 , equation (19) has a non-oscillatory solution. If

$$
\lim _{t \rightarrow \infty} \inf \tau_{3}\left(a+b \sin t+c \sin \frac{t}{2}\right)>\frac{1}{e}
$$

then, by Corollary 1, all solutions of equation (19) are oscillatory.

\section{REFERENCES}

[1] Yongshao Chen, 'Oscillation and asymptotic behavior of solutions of first order linear functional differential equations with oscillatory coefficients', (in Chinese), Acta Math. Appl. Sinica 12 (1989), 96-104.

[2] I. Györi and G. Ladas, Oscillation theory of delay differential equations with applications (Clarendon Press, Oxford, 1991).

[3] B.R. Hunt and J.A. Yorke, 'When all solutions of $x^{\prime}=-\sum q_{i}(t) x\left(t-\tau_{i}(t)\right)$ oscillate', $J$. Differential Equations 53 (1984), 139-145.

[4] R.G. Koplatadze and T.A. Chanturia, 'On the oscillatory and monotone solutions of first order differential equations with deviating arguments', (in Russian), Differensial'nye Uravneniya 18 (1982), 1463-1465.

[5] M.R. Kulenovic and M.K. Grammatikopoulos, 'First order functional differential inequalities with oscillating coefficients', Nonlinear Anal. 8 (1984), 1043-1054.

[6] M.R. Kulenovic and M.K. Grammatikopoulos, 'Some comparison and oscillation results for first order differential equations and inequalities with a deviating argument', J. Math. Anal. Appl. 131 (1988), 67-84.

[7] M.K. Kwong, 'Oscillation of first order delay equations', J. Math. Anal. Appl. 156 (1991), 274-286.

[8] G. Ladas, Y.G. Sficas and I.P. Stavroulakis, 'Functional differential inequalities and equations with oscillating coefficients', in Trends in theory and practice of nonlinear differential equations, (V. Lakshmikantham, Editor) (Marcel Dekker, New York, Basel, 1984). 
[9] J.S. Yu, Z.C. Wang, B.G. Zhang and X.Z. Qian, 'Oscillations of differential equations with deviating arguments', Pan. Math. J. 2 (1992), 59-78.

Department of Information

Shanxi Economic Management Institute

Taiyuan, Shanxi 030006

People's Republic of China

(Chunhai Kou)

Department of Mathematics

Shanxi University

Taiyuan, Shandi 030006

People's Republic of China
Department of Mathematics

Shanri University

Taiyuan, Shanri 030006

People's Republic of China

(Weiping Yan and Jurang Yan) 